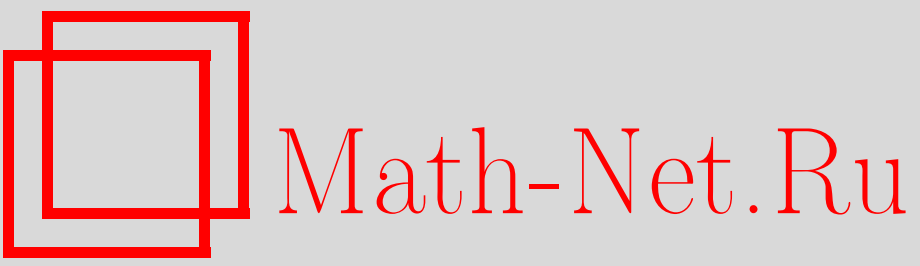

А. М. Райгородский, О хроматическом числе пространства с метрикой $l_{q}, \quad \mathrm{MH}$, 2004, том 59, выпуск 5, 161-162

DOI: https://doi.org/10.4213/rm784

Использование Общероссийского математического портала Math-Net.Ru подразумевает, что вы прочитали и согласны с пользовательским соглашением

http://www.mathnet.ru/rus/agreement

Параметры загрузки:

IP : 54.81 .137 .203

26 апреля 2023 г., 15:18:24 


\section{О ХРОМАТИЧЕСКОМ ЧИСЛЕ ПРОСТРАНСТВА С МЕТРИКОЙ $l_{q}$}

\section{А. М. РАЙГОРОДСКИй}

1. Введение и формулировка результата. В своей работе (см. [1] и [2]), которая была весьма популярна среди специалистов и тем не менее в течение четверти века оставалась неопубликованной, М. Бенда и М. Перлес ввели понятие хроматического числа метрического пространства, определяемого следующим образом. Пусть $(X, \rho)$ - это некоторое метрическое пространство с метрикой $\rho$. Зафиксируем произвольное положительное вещественное число $b$ и рассмотрим граф $\mathscr{G}=(\mathscr{V}, \mathscr{E})$, у которого множество вершин $\mathscr{V}$ совпадает с $X$, а ребрами соединены те и только те пары вершин $x, y \in X$, расстояние между которыми равно $b: \rho(x, y)=b$. Тогда расстояние $b$ называется "запрещенным" или "критическим", а стандартное хроматическое число $\chi(\mathscr{G})$ графа $\mathscr{G}$ - хроматическим числом пространства $(X, \rho)$ с этим запретом. Величину $\chi(\mathscr{G})$ принято обозначать, подчеркивая ее геометрическую природу, через $\chi((X, \rho), b)$. В существенной степени деятельность Бенды и Перлеса была мотивирована попытками подобраться к решению классической проблемы П. Эрдёша и Г. Хадвигера (см. [3]) об отыскании хроматического числа ариффметического пространства $\mathbb{R}^{n}$, снабженного евклидовой метрикой. Однако к настоящему времени общая постановка вопроса носит практически столь же классический характер.

Здесь мы изучим два класса метрических пространств: класс $\left(\mathbb{R}^{n}, l_{q}\right)$ и класс $\left(\mathbb{Q}^{n}, l_{q}\right)$ (под $\mathbb{Q}^{n}$ мы понимаем множество всех $n$ - мерных векторов с рациональными координатами, а под $l_{q}$ - метрику вида $l_{q}(\mathbf{x}, \mathbf{y})=\left(\left|x_{1}-y_{1}\right|^{q}+\cdots+\left|x_{n}-y_{n}\right|^{q}\right)^{1 / q}, l_{\infty}(\mathbf{x}, \mathbf{y})=\max _{i}\left|x_{i}-y_{i}\right|$, $\left.\mathbf{x}=\left(x_{1}, \ldots, x_{n}\right), \mathbf{y}=\left(y_{1}, \ldots, y_{n}\right)\right)$. Нас будут интересовать оценки соответствующих хроматических чисел при условии неограниченного роста размерности. Для начала мы выпишем вкратце известные результаты. В случае с $\left(\mathbb{R}^{n}, l_{2}\right)$ мы имеем дело с задачей Эрдёша-Хадвигера, причем здесь значение критического расстояния не играет никакой роли: для всякого $b$ выполнены неравенства $(1.239 \ldots+o(1))^{n} \leqslant \chi\left(\left(\mathbb{R}^{n}, l_{2}\right), b\right) \leqslant(3+o(1))^{n}$ (нижняя оценка принадлежит автору [4], а верхняя - Д. Ларману и К.А. Роджерсу [5]). Для $\mathbb{Q}^{n}$ с той же, евклидовой, метрикой доказаны чуть более слабые утверждения: $\chi\left(\left(\mathbb{Q}^{n}, l_{2}\right), b\right) \geqslant(1.173 \ldots+o(1))^{n}$, $\chi\left(\left(\mathbb{Q}^{n}, l_{2}\right), b\right) \geqslant \Omega\left((1.239 \ldots+o(1))^{n}\right)$, где $b \in \mathbb{Q}, \chi\left(\left(\mathbb{Q}^{n}, l_{2}\right), b\right) \geqslant(1.239 \ldots+o(1))^{n}$ для подходящих $b \in \mathbb{R} \backslash \mathbb{Q}($ см. $[6])$ и $\chi\left(\left(\mathbb{Q}^{n}, l_{2}\right), b\right) \leqslant \chi\left(\left(\mathbb{R}^{n}, l_{2}\right), b\right) \leqslant(3+o(1))^{n}$. При $q=\infty$ легко убедиться в том, что оба наших хроматических числа равны $2^{n}$, коль скоро, например, $b=1$. Наконец, при любом $q<\infty$ Дж.-Х. Канг и З. Фюреди установили недавно оценку $\chi\left(\left(\mathbb{R}^{n}, l_{q}\right), b\right) \leqslant(5+o(1))^{n}$, а тот факт, что $\chi\left(\left(\mathbb{R}^{n}, l_{q}\right), b\right) \geqslant(1.207 \ldots+o(1))^{n}$ непосредственно вытекает из работы $\Pi$. Франкла и Р. Уилсона [7]. Ясно, что, в свою очередь, $\chi\left(\left(\mathbb{Q}^{n}, l_{q}\right), b\right)$ ограничено сверху правой частью оценки Канга-Фюреди при каждом $q$ и $b$. Что же касается нижней оценки для $\chi\left(\left(\mathbb{Q}^{n}, l_{q}\right), b\right)$, то сразу можно сказать толњко, что для любого $q$ найдутся $b$, при которых она совпадает $c$ неравенством Франкла-Уилсона. Прежде чем переходить теперь к формулировке основного резултата, заметим, что более подробный обзор по проблеме может быть найден в статье автора [6].

Tеорема 1. 1. Для всякого $X \in\left\{\mathbb{R}^{n}, \mathbb{Q}^{n}\right\}$ и для всякого $b\left(b \in \mathbb{R}\right.$, если $X=\mathbb{R}^{n}, u$ $b \in \mathbb{Q}$, если $X=\mathbb{Q}^{n}$ ) выполнена оценка

$$
\chi\left(\left(X, l_{1}\right), b\right) \geqslant \max _{a \in\left(0, \frac{1}{2}\right)} \frac{C_{n}^{\left[a n{ }_{2}\right.}{ }^{[a n]}}{Q_{1}(n) C_{n}^{[a n / 4]} C_{n-[a n / 4]}^{[a n / 4]}}=(1.365 \ldots+o(1))^{n} .
$$

Здесь $Q_{1}(n)$ - некоторая функция, растущая медленнее любой экспоненть, а максимум достигается при $a=(6-\sqrt{12}) / 6$.

2. Пусть $q \in \mathbb{N}$. Тогда при любом $b \in \mathbb{Q}$ виполнена оченка

$$
\chi\left(\left(\mathbb{Q}^{n}, l_{q}\right), b\right) \geqslant \min _{u \in\left(\frac{1}{2^{q+1}}, \frac{1}{2}\right)} \frac{C_{n}^{[u n]}}{Q_{2}(n) C_{n}^{[u n / 2]}}=(1+\varepsilon+o(1))^{n},
$$

где $Q_{2}(n)$ - субэкспоненциальная функция, $a \varepsilon=\varepsilon(q)>0$.

2. Эскиз доказательства теоремы 1. В этом параграфе мы по пунктам изложим основные шаги, необходимые нам для доказательства первой части теоремы. В действительности,

Работа выполнена при финансовой поддержке Российского фонда фундаментальных исследований (грант № 02-01-00912), гранта поддержки Ведущих научных школ НШ-136.2003.1, гранта Президента РФ МК-3130.2004.1 и гранта ИНТАС 03-51-5070. 
рассуждения, обосновьвающие вторую часть нашего утверждения, в существенной степени параллельны нижеследующим, и в данной заметке мы на них не останавливаемся.

1. Пусть $a \in\left(0, \frac{1}{2}\right)$. Рассмотрим $V_{a}=\left\{\mathbf{x}=\left(x_{1}, \ldots, x_{n}\right) \in\{-1,0,1\}^{n}: \operatorname{card}\{i=1, \ldots, n:\right.$ $\left.\left.x_{i}= \pm 1\right\}=[a n]\right\} \subset \mathbb{Q}^{n} \subset \mathbb{R}^{n}$. Ясно, что card $V_{a}=C_{n}^{[a n]} 2^{[a n]}$. Пусть $p-$ минимальное простое число, удовлетворяющее соотношению $2 p>[a n]$, так что заведомо $p=p(n)$ асимптотически приближается к $[a n] / 2$ (см. [8]). Образуем граф̆ $G_{a}=\left(V_{a}, E_{a}\right)$, полагая $E_{a}=\{(\mathbf{x}, \mathbf{y}) \in$ $\left.V_{a} \times V_{a}: l_{1}(\mathbf{x}, \mathbf{y})=2 p\right\}$.

2. С помощью довольно тонкой модификации линейно-алгебраического метода в комбинаторике (cp. [4], [6], [7] и [9]) можно показать, исходя из свойств $p$, что число независимости графа $G_{a}$ (см. [10]) оценивается сверху величиной $Q_{1}(n) C_{n}^{[a n / 4]} C_{n-[a n / 4]}^{[a n / 4]}$. Стало быть, автоматически $\chi\left(G_{a}\right) \geqslant \frac{C_{n}^{[a n]}{ }_{2}^{[a n]}}{Q_{1}(n) C_{n}^{[a n / 4]} C_{n-[a n / 4]}^{[a n / 4]}}$.

3. Пусть $\mathscr{G}$ - граф̆, через посредство которого мы определяли величину $\chi\left(\left(X, l_{1}\right), 2 p\right), X \in$ $\left\{\mathbb{R}^{n}, \mathbb{Q}^{n}\right\}$. Понятно, что $G_{a} \subset \mathscr{G}$, и, значит,

$$
\chi\left(\left(X, l_{1}\right), 2 p\right)=\chi(\mathscr{G}) \geqslant \chi\left(G_{a}\right) \geqslant \frac{C_{n}^{[a n]} 2^{[a n]}}{Q_{1}(n) C_{n}^{[a n / 4]} C_{n-[a n / 4]}^{[a n / 4]}} .
$$

Неравенство выполнено для каждого $a$, в силу чего мы вольны взять в его правой части максимум по $a \in\left(0, \frac{1}{2}\right)$. Таким образом, при $b=2 p$ утверждение доказано. Однако, за счет свойств наших метрических пространств, мы теперь имеем право нормировать всю конструкцию графа $G_{a}$ в случае $\mathbb{R}^{n}$ на произвольное вещественное, а в случае $\mathbb{Q}^{n}$ на произвольное рациональное число, что и завершает эскиз доказательства.

3. Дополнение. Пусть $(X, \rho)$ - метрическое пространство, а $\mathscr{G}=(\mathscr{V}, \mathscr{E})$, где $\mathscr{V}=X, \mathscr{E}=$ $\left\{(x, y) \in X \times X: \rho(x, y) \in\left\{b_{1}, \ldots, b_{r}\right\}\right\}, b_{1}>0, \ldots, b_{r}>0$. Положим $\chi\left((X, \rho), b_{1}, \ldots, b_{r}\right)=$ $\chi(\mathscr{G})$. Задача об отыскании таких хроматических чисел восходит к П. Эрдёшу, которьй рассматривал пространство $\left(\mathbb{R}^{2}, l_{2}\right)$ и установил оценку $\max _{b_{1}, \ldots, b_{r}} \chi\left(\left(\mathbb{R}^{2}, l_{2}\right), b_{1}, \ldots, b_{r}\right) \geqslant$ $c r \sqrt{\log r}, c>0$. В случае, когда $(X, \rho)=\left(\mathbb{R}^{n}, l_{2}\right)$, задача изучалась автором в $[6]$, где, в частности, обоснованы неравенства $\max _{b_{1}, b_{2}} \chi\left(\left(\mathbb{R}^{n}, l_{2}\right), b_{1}, b_{2}\right) \geqslant(1.439 \ldots+o(1))^{n}$ и $(\nu r)^{\mu n} \leqslant$ $\max _{b_{1}, \ldots, b_{r}} \chi\left(\left(\mathbb{R}^{n}, l_{2}\right), b_{1}, \ldots, b_{r}\right) \leqslant(3+o(1))^{r n}, \nu>0, \mu>0$. Благодаря разработанной нами технике (модификация линейно-алгебраического метода, упомянутая в пункте 2 предыдущего параграфа), удается доказать следующую новую теорему.

ТЕорема 2. Виполнены оценки $\max _{b_{1}, b_{2}} \chi\left(\left(\mathbb{R}^{n}, l_{1}\right), b_{1}, b_{2}\right) \geqslant(1.607 \ldots+o(1))^{n},\left(\nu^{\prime} r\right)^{\mu^{\prime} n}$ $\leqslant \max _{b_{1}, \ldots, b_{r}} \chi\left(\left(\mathbb{R}^{n}, l_{1}\right), b_{1}, \ldots, b_{r}\right) \leqslant(5+o(1))^{r n}$, где $\nu>0, \mu>0$ - абсолютные конcтанты.

Отметим, что верхняя оценка в последней серии неравенств мгновенно следует из упоминавшейся в первом параграфе работы Канга и Фюреди.

\section{СПИСОК ЛИТЕРАТУРЫ}

[1] P. D. Johnson, Jr.// Geombinatorics. 2000. V.9. P. 110-112. [2] M. Benda, M. Perles // Geombinatorics. 2000. V. 9. P. 113-126. [3] H. Hadwiger // Portugal. Math. 1944. V. 4. P. 140-144. [4] А. М. Райгородский // УМН. 2000. Т. 55. № 2. С. 147-148. [5] D. G. Larman, C. A. Rogers // Mathematika. 1972. V. 19. P. 1-24. [6] A. М. Райгородский // УМН. 2001. T. 56. № 1. C. 107-146. [7] P. Frankl, R. M. Wilson // Combinatorica. 1981. V. 1. P. 357-368. [8] К. Прахар. Распределение простых чисел. М.: Мир, 1967. [9] L. Babai, P. Frankl. Linear algebra methods in combinatorics. Part 1. Preliminary version 2. Chicago: Department of Computer Science, Univ. Chicago, 1992. [10] Ф. Харари. Теория графов. М.: Мир, 1973. 